\title{
BMJ Open Independent prescribing by advanced physiotherapists for patients with low back pain in primary care: protocol for a feasibility trial with an embedded qualitative component
}

\author{
Timothy David Noblet, ${ }^{1,2}$ John F Marriott, ${ }^{3}$ Alison B Rushton ${ }^{\oplus 1}$
}

To cite: Noblet TD, Marriott JF, Rushton AB. Independent prescribing by advanced physiotherapists for patients with low back pain in primary care: protocol for a feasibility trial with an embedded qualitative component. BMJ Open 2019;9:e027745. doi:10.1136/ bmjopen-2018-027745

- Prepublication history and additional material for this paper are available online. To view these files, please visit the journal online (http://dx.doi org/10.1136/bmjopen-2018027745).

Received 5 November 2018

Revised 7 March 2019

Accepted 12 March 2019

Check for updates

(C) Author(s) (or their employer(s)) 2019. Re-use permitted under CC BY-NC. No commercial re-use. See rights and permissions. Published by BMJ.

${ }^{1}$ CPR Spine, School of Sports, Exercise and Rehabilitation Sciences, University of Birmingham, Birmingham, UK

${ }^{2}$ Faculty of Medicine and Health Sciences, Macquarie University, Sydney, New South Wales,

Australia

${ }^{3}$ School of Health and Population Sciences, College of Medical and Dental Sciences, University of Birmingham, Birmingham, UK

Correspondence to

Mr Timothy David Noblet; tdn818@bham.ac.uk

\section{ABSTRACT}

Introduction Low back pain (LBP) is the most prevalent musculoskeletal condition in the UK. Guidelines advocate a multimodal approach, including prescription of medications. Advanced physiotherapy practitioners (APPs) are well placed to provide this care in primary care. Physiotherapist independent prescribing remains novel, with the first prescribers qualifying in 2014. This feasibility trial aims to evaluate the feasibility, suitability and acceptability of assessing the effectiveness of independent prescribing by APPs for patients with LBP in primary care, to inform the design of a future definitive stepped-wedged cluster trial.

Method and analysis (1) Trial component. An APP (registered prescriber) will complete the initial participant consultation. If prescription drugs are required within the multimodal physiotherapeutic context, these will be prescribed. Patient-reported outcome measures will be completed prior to initial assessment and at 6 and 12 weeks to assess feasibility of follow-up and data collection procedures. Accelerometers will be fitted for 7 days to assess physical activity, sedentary behaviour and feasibility of use. (2) Embedded qualitative component. A focus group and semistructured interviews will be used to evaluate the views and experiences of the participants and APPs respectively, about the feasibility, suitability and acceptability of the proposed full trial. A Consolidated Standards of Reporting Trials diagram will be used to analyse feasible eligibility, recruitment and follow-up rates. Descriptive analysis of the data will be completed to evaluate procedures. Thematic analysis will be used to analyse and synthesise the qualitative data.

Ethics and dissemination This feasibility trial is approved by the Health Research Authority (HRA). Ethical approval was sought and granted via the Integrated Research Application System (IRAS) ID 250734. Data will be disseminated via publication in peer reviewed journal and conference presentation. It is anticipated that the results of this study will be used in conjunction with ethical evaluation, economic and risk analyses, as well as consultation with key stakeholders including the British health consumer when contemplating change, enhancement or redesign of the essential full randomised controlled trial.

Trial registration number ISRCTN15516596, Pre-results.
Strengths and limitations of this study

- First rigorous investigation aiming to evaluate the methods required to assess the clinical and cost-effectiveness of independent prescribing by advanced physiotherapists for patients with low back pain in primary care.

- The design of this feasibility trial was developed by clinicians, academics, methodological experts, healthcare service managers, professional leaders and the public/patients.

- The methods will be tested across a range of cities, towns and villages in varying geographical areas across England.

\section{BACKGROUND}

Low back pain (LBP) is the most prevalent musculoskeletal condition in the UK, with $58 \%-84 \%$ of the population experiencing LBP in their lifetime. ${ }^{1-3}$ At any time, $28.5 \%$ of adults $>25$ years are experiencing LBP. ${ }^{2}$ Data indicate that 3.2 million work days are lost per year in the UK, with an average of 16.5 days lost per case. ${ }^{4}$ Approximately $20 \%$ of those with LBP seek care from their general practitioner (GP), ${ }^{1}$ with $7 \%$ of all GP consultations being due to LBP. ${ }^{35}$

Despite increased funding for treatments and a growing understanding of the complex biopsychosocial nature of LBP leading to improvements in assessment and management of the condition, up to $7 \%$ of the general population in the UK have chronic LBP associated with significant disability $^{12}$ and the health and function of this demographic continues to decline. ${ }^{6}$ In an attempt to address this, novel approaches have been adopted to inform shared decision-making, and stratification tools are being used to improve outcomes through recognising clinical heterogeneity, ensuring that 
all biopsychosocial risk factors are addressed, improving patient management and reducing the overall cost of healthcare ${ }^{6-8}$ Early assessment, diagnosis and treatment of LBP has been seen to reduce chronicity. ${ }^{1}$ However, the complex and multidimensional nature of LBP combined with a current deficit in the availability of GPs in the UK ${ }^{910}$ has prompted the redesign of outdated traditional LBP clinical pathways, and the introduction of new treatment models designed to maximise clinical and cost-effectiveness, while readying the health services for the future. ${ }^{10-12}$

Physiotherapists are experts in the assessment, diagnosis and treatment of musculoskeletal disorders. ${ }^{13}$ For $>30$ years, physiotherapists have been working in advanced practice roles across the country, using their scope of practice to optimise patient care, providing support in health services where the availability of medical practitioners does not meet the demands of a local community. ${ }^{13} 14$ Advanced musculoskeletal physiotherapists have been shown to be clinically and cost-effective when working in a variety of settings including orthopaedic and emergency care departments as well as in primary care in musculoskeletal interface services. ${ }^{14-16}$ Recently, the success and experience of these practitioners, alongside changes in demographics and predictions that GP numbers will further reduce by 2020 , have prompted successful pilot studies investigating the effectiveness of first contact advanced physiotherapy practitioners (FCPs) in primary care ${ }^{11}{ }^{17}$ As a result, Health Education England, in collaboration with NHS England, the Royal College of General Practitioners (RCGP), the British Medical Association and the Chartered Society of Physiotherapy have committed to introducing these roles across England. ${ }^{17-19}$

Recently published guidelines from the National Institute for Health and Care Excellence (NICE) ${ }^{8}$ for LBP and sciatica, advocate for a holistic, multimodal approach to assessment and management. ${ }^{3}$ Advanced physiotherapists are well placed to provide this care owing to their competency in physical therapies including manual and exercise therapy; knowledge and skills associated with the management of psychosocial factors and ability to appropriately refer for blood tests, imaging, spinal injections, denervation and surgery. ${ }^{20}{ }^{21}$ Further, the NICE guidelines recommend the use of drugs that are helpful and minimise harm. ${ }^{38}$ It is therefore envisaged that independent physiotherapist prescribing will be a key competency required for the successful implementation of first contact advanced physiotherapists working in primary care.

Independent physiotherapist prescribing remains relatively new, with the first prescribers qualifying in 2014. Evaluation of physiotherapist and podiatrist independent prescribing has shown good acceptance by patients and a good safety record to date..$^{22} \mathrm{~A}$ recent mixed-methods systematic review of investigating the barriers and facilitators of non-medical prescribing (NMP) concludes that the successful implementation and utilisation of NMP is dependent on adequate preparation and organisation of a range of factors. ${ }^{23}$ Considerations such as the use of advanced physiotherapists in primary care were seen to facilitate successful implementation of NMP as long as clinical governance, policy development and service practicalities and logistics are adequately developed and established prior to implementing NMP. To ensure longevity and future growth, education, support and financial factors alongside the management of personal and professional considerations were also deemed paramount. ${ }^{23}$

For clinical services to be successful, they must deliver positive clinical outcomes in a safe and economically sound manner. ${ }^{24}$ Our recent rigorous systematic review investigating the clinical and cost-effectiveness of NMP across all professions and clinical settings, identified limited evidence with unclear risk of bias. ${ }^{25}$ We concluded that quantifiable benefits of NMP remain unknown and called for adequately powered, low risk of bias randomised controlled trials (RCTs) in specific patient groups, professions and clinical settings. ${ }^{25}$ Owing to the contemporary nature of independent physiotherapist prescribing, no trial has examined the clinical or cost-effectiveness of this intervention in the complex context of LBP. Trial design required careful consideration, particularly, as independent physiotherapy prescribing is within the process of implementation across private health services and NHS Trusts. A feasibility study is therefore required to inform a multicentre RCT investigating physiotherapist independent prescribing by advanced physiotherapists for patients with LBP, in primary care. The project will aim to evaluate the feasibility, suitability and acceptability of procedures and outcomes for use in the full trial, also assessing the commitment and burden on participants, clinicians and researchers as well as infrastructure and technological requirements.

\section{AIM}

To evaluate the feasibility, suitability and acceptability of assessing the effectiveness of independent prescribing by advanced physiotherapy practitioners (APPs) for patients with LBP in primary care to inform the design of a future definitive stepped-wedged cluster trial.

\section{OBJECTIVES}

\section{General objectives}

- To assess the feasibility, suitability and acceptability of the proposed full trial ${ }^{26}$ including the following:

- Eligibility criteria. ${ }^{27-29}$

- Recruitment strategy. ${ }^{27-29}$

- Data collection methods. ${ }^{27-29}$

- Follow-up procedures. ${ }^{27} 28$

\section{Specific objectives}

Feasibility

- To evaluate participant recruitment rates. ${ }^{26-28}$

- To evaluate the ease of fitting participants with accelerometers and ease of data collection. ${ }^{27} 28$ 
- To evaluate the capacity (time and effort) of clinicians and researchers to complete trial-related tasks. ${ }^{27} 28$

- To evaluate the necessary training required by clinicians to successfully implement a full trial. ${ }^{27} 28$

\section{Suitability}

- To evaluate the range of participants' scores on the Roland and Morris Disability Questionnaire (RMDQ), assessing for floor effects and therefore the appropriateness of outcome measure for use in a full trial. ${ }^{26-29}$

- To evaluate participant compliance with wearing the accelerometer device. ${ }^{27} 28$

- To evaluate the time required to conduct each stage of the protocol. ${ }^{27} 28$

- To evaluate the appropriateness and availability of services and infrastructure such as access to national and institutional communication and information technologies required to undertake a full trial. ${ }^{27} 28$

\section{Acceptability}

- To evaluate the acceptability of the intervention to patients and the public. ${ }^{26-29}$

\section{METHODS}

To ensure transparency and reproducibility, this feasibility trial protocol has been registered on the ISRCTN database and is reported in line with the CONSORT 2010 statement: extension to randomised pilot and feasibility trials, ${ }^{30-32}$ with all patient and public involvement (PPI) reported in line with the GRIPP2 short form reporting checklist. $^{33} 34$

The feasibility trial will use a mixed-methods research approach, comprising the following:

- A quantitative one-armed feasibility trial.

- Qualitative semistructured interviews and patient focus groups, using thematic analysis.
Mixed-methods designs are recognised to enable a richer synthesis, generating data that will facilitate appropriate change. ${ }^{35-37}$

\section{Design}

RCTs are considered the gold standard for evaluating the effectiveness of an intervention. ${ }^{38}$ Cluster RCTs (cRCTs) allowing for randomisation by group have been developed to overcome practical issues in clinical settings, where individual randomisation is not convenient or feasible. ${ }^{38-40}$ When evaluating contemporary interventions, parallel deigns requiring the new intervention to be simultaneously provided to multiple clusters of participants are often too costly or not practical owing to the necessary clinician training required to deliver the intervention safely. ${ }^{38}$ A stepped-wedge cluster randomised controlled trial (SWcRCT) design will therefore be used to evaluate the clinical and cost-effectiveness of physiotherapist prescribing for LBP in the future. This design is valuable when evaluating innovative clinical interventions where there is a strong ethical belief that the intervention will benefit patients. ${ }^{39} 4142$ SWcRCTs allow each experimental cluster to begin in the control arm then cross over to the experimental arm at specified time points (figure 1). ${ }^{41}$ As the implementation of independent physiotherapy prescribing and the utilisation of APPs working as FCPs are both relatively contemporary innovations, there are limited numbers of clinicians currently working in these innovative roles who are registered to prescribe. This research design allows for the use of fewer clinicians than those required for a parallel design and is therefore more reflective of current practice. APPs who are not prescribers will start in the control group and cross to the experimental group following registration as an independent prescriber. APPs who are not prescribers start in the control group and cross to the experimental group. ${ }^{39-42}$

Figure 1 The SWCRCT design for potential use in a full trial. APP, advanced physiotherapy practitioner; FCP, first contact advanced physiotherapy practitioner; SWcRCT, stepped-wedge cluster randomised controlled trial. 
Currently, no clear framework exists describing the requirements for best practice when completing feasibility trials in preparation for SWcRCTs. ${ }^{43}$ Two-arm feasibility trials that have aimed to calculate intracluster correlation coefficients required for sample size calculations in preparation for full cRCTs have demonstrated insufficient accuracy, unless the feasibility trial is equal in size to the proposed full trial. ${ }^{43}$ Therefore, a single-arm feasibility design will be employed to test specific aspects of the trial protocol in terms of feasibility, suitability and acceptability on the experimental arm of the future SWCRCT, without sample size estimation. ${ }^{27} 4445$

\section{Trial component}

A prospective, mixed-methods, single-group feasibility trial will be used to evaluate the trial objectives. ${ }^{294}$ Participant consent forms (online supplementary file 1) and patient-reported outcome measures (online supplementary file 2) will be completed digitally via an online survey at initial assessment (baseline) and at 6 and 12 weeks (12 weeks is the planned primary end point of the definitive trial) following a prescription being issued, to evaluate the feasibility of follow-up data collection procedure ${ }^{45}{ }^{46}$ Follow-up time points have been selected in line with the prognostic literature showing that $40 \%$ of patients presenting to primary care with LBP will be pain-free 6 weeks post onset, with $58 \%$ pain-free by 12 weeks. ${ }^{47-49}$ The online outcome measures survey will be built using REDCap (Research Electronic Data Capture) software (hosted in the Centre for Precision Rehabilitation for Spinal Pain [CPR Spine] at the University of Birmingham, UK), enabling data to be captured and stored in real time, on a range of electronic devices. ${ }^{50}$ Baseline measurements will be completed by the participants within the clinical setting. A link to the online outcome measures survey with instructions will be emailed to participants for completion at 6 and 12 weeks. If participants forget to complete the outcome questionnaire on the required day, a reminder to complete it will be sent at 24 and 48 hours after the deadline to facilitate compliance. ${ }^{451}$ To evaluate the feasibility of fitting participants with accelerometers in clinic, the ease of data collection and participant compliance with wearing the accelerometer device, ${ }^{2728} \mathrm{n}=10$ participants at one research site will be fitted with an accelerometer to wear for 7 days immediately following completion of patient-reported outcome measures at the first consultation. Participants will be provided with stamped/addressed envelopes in which to return the devices after use.

\section{Participants}

Potential participants will be identified by the APPs at each clinical site, by using the STarT Back Tool at initial assessment, to stratify all patients presenting with LBP. $^{7}$ Patients stratified into the medium risk group by the STarT Back Tool will be eligible for recruitment if they meet the inclusion criteria following assessment (box 1). This group of patients have been recognised

\section{Box 1 Participant eligibility criteria}

\section{Inclusion Criteria}

- Male and female patients, aged >18 years.

- Non-specific LBP with or without leg pain requiring medication advice and drug prescription on assessment.

- Classified as moderate risk using the STarT Back Tool (classified as potentially benefiting from medicines and active physiotherapy treatment ${ }^{7}$ ).

- Able to read/communicate in English (owing to funding restrictions for interpreters and translators).

- Capable of following the demands inherent of the study.

\section{Exclusion Criteria}

- Signs of lumbar nerve root compression. ${ }^{82}$

- Red Flags including potential spinal fracture, inflammatory disease, infection or malignancy. ${ }^{82}$

- Spinal stenosis. ${ }^{83}$

- Suspicion of or confirmed cauda equina syndrome. ${ }^{84}$

Does not have capacity to consent. ${ }^{85}$

- Unable to receive email and/or complete online questionnaires.

LBP, low back pain.LBP, low back pain.

as predominant cohort presenting for assessment and treatment of LBP in primary care; exhibiting both physical and psychosocial prognostic factors and may require physiotherapist prescribing to optimise their multimodal physiotherapeutic treatment. ${ }^{752-54}$ Convenience sampling will be adopted, as this method has the advantages of fluid recruitment and follow-up required by feasibility trials, with good retention of participants where time is limited..$^{28} 454655$ Patients who are interested in participating will be provided with a participant information sheet (online supplementary file 3) explaining the rationale, content and research dissemination plans to ensure ethical recruitment of participants. The physiotherapist will answer any questions and if the patient wishes to participate, consent will be obtained using an online consent form. Contact details for the research team will be provided to give the participants the opportunity to have any further questions answered. Contact details for an independent advisory service (PALS at each site) will also be provided in case external advice is desired by participants. Participants will be free to withdraw at any time, without any impact on their care. ${ }^{45} 46$

\section{Interventions}

As the control arm of the definitive trial will be "current normal practice', the intervention designed for the experimental arm of the definitive trial will be used to evaluate the feasibility trial objectives. ${ }^{26-29}$ As per 'current normal practice', an APP acting as an FCP will complete the initial assessment and physiotherapeutic treatment of participants as deemed appropriate through evidence-based clinical reasoning and best practice (traditional role). In addition to the physiotherapist's traditional role, the APP will have the competence and legal ability to prescribe medicines independently. If advice about medication or prescription drugs are required/no longer required 
Table 1 Secondary outcome measures and their rationale

\begin{tabular}{lll}
\hline Outcome & Measure & Rationale \\
\hline $\begin{array}{l}\text { Health-related quality of } \\
\text { life (QALY) }\end{array}$ & EQ-5D 5L & $\begin{array}{l}\text { The EQ-5D } 5 \mathrm{~L} \text { is used to measure health-related quality of life demonstrating good reliability and } \\
\text { validity through psychometric testing. } \\
\text { a full } \text { If feasibility is found, this measure will inform cost utility in }\end{array}$ \\
$\begin{array}{l}\text { Pain-related fear of } \\
\text { movement }\end{array}$ & $\begin{array}{l}\text { The TSK } \\
\text { The TSK is a 17-item tool that was developed to measure a person's fear of movement owing } \\
\text { to LBP. Ongoing fear of movement has been linked to the development of long-term persistent } \\
\text { pain. } \\
\text { measuring pain-related fear of movement. }\end{array}{ }^{88}$ This to show good validity and reliability when
\end{tabular}

Physical activity and ActivPal 3 Accelerometer Anecdotal evidence suggests that decreasing sedentary behaviour in people with LBP may have significant health benefits, ${ }^{53}$ reducing risks of obesity, metabolic syndrome, type 2 diabetes and mortality. ${ }^{89}$ Systematic reviews have revealed that physical activity of people with LBP is lower or equal to the healthy population ${ }^{90-92}$; however, there appears to be differing patterns of physical behaviour, with the back-pain population engaging in shorter bouts of physical activity that are not long enough to incur health benefits $(>10 \mathrm{~min}) \cdot{ }^{92}{ }^{93}$ An accelerometer will be used to collect data including steps count and sedentary periods. ${ }^{94}$ To date, no individual brand/ model of accelerometer has been identified as gold standard. The ActivPal 3 has been selected for use in this feasibility trial as it has been seen to be more precise and sensitive than other accelerometers. $^{9495}$

ActivPal 3 Accelerometer $50 \%-60 \%$ of people experiencing either acute or persistent LBP experience high levels of sleep disturbance. ${ }^{96}$ Poor sleep over long periods of time may lead to depression, obesity, diabetes and cardiovascular disease. ${ }^{969}$ Patients with LBP suffering with sleep disturbance have been reported as twice as likely to be hospitalised. ${ }^{98}$ Improved sleep has been seen to modulate pain intensity, ${ }^{99}$ with poor quality sleep associated with increased pain intensity, fatigue, decreased function and psychological stress. An accelerometer will be used to collect sleep duration data alongside physical activity and sedentary behaviour. ${ }^{100}$

\begin{tabular}{lll}
$\begin{array}{l}\text { Time to return to work } \\
\text { and nature of return to } \\
\text { work (eg, full time, part } \\
\text { time and light duties) }\end{array}$ & Days & $\begin{array}{l}\text { Work absence owing to sick leave for work disability is a key issue clinically, socially and } \\
\text { economically. The minimally clinically important change (MCIC) for time to return to work has } \\
\text { not been defined due to the specific measurement (days on sick leave) being widely accepted } \\
\text { and recognition of the measure's value in social and economic issues rather than an indicator of } \\
\text { morbidity. }{ }^{62} \text { This measure would therefore be useful when conducting economic evaluation of } \\
\text { physiotherapist prescribing. }\end{array}$ \\
$\begin{array}{l}\text { Prescription utilisation, } \\
\text { participant }\end{array}$ & Days & $\begin{array}{l}\text { Time requiring drugs for the treatment of non-specific LBP discussed/prescribed by the advanced } \\
\text { physiotherapists will be monitored to evaluate the necessity of this measure for future cost- } \\
\text { effectiveness analysis within a full trial. }\end{array}$ \\
\hline $\begin{array}{l}\text { Number of appointments } \\
\text { with other healthcare } \\
\text { professionals about this } \\
\text { episode of LBP }\end{array}$ & $\begin{array}{l}\text { Number of appointments } \\
\text { with each type of } \\
\text { healthcare professional }\end{array}$ & $\begin{array}{l}\text { The number of appointments with other healthcare professionals about the specific episode of } \\
\text { LBP being studied will be recorded via a question in the outcome questionnaire to evaluate the } \\
\text { necessity of this measure for future cost-effectiveness analysis within a full trial. }\end{array}$ \\
\hline
\end{tabular}

within the multimodal physiotherapeutic context, these will be prescribed/de-prescribed by the APP immediately, rather than referring the patient back to their GP for assessment for medications as per current normal practice. The medications provided should be taken by the patient as prescribed in the time frames discussed in the clinical consultation.

\section{Outcomes}

The literature reports that the use of a core outcome set assessing pain intensity, health-related quality of life and physical function is required for the assessment of non-specific LBP. ${ }^{56}$ However, no consensus exists with regards to the instruments most suitable to measure these domains. ${ }^{56}$ The outcome measures selected for use within the trial were informed by a team of subject-experts including physiotherapists, pharmacists, medical practitioners, academics and health-service managers and deemed most appropriate to evaluate the study's objectives while attempting to minimise the burden on participants. Two primary outcome measures (detailed below) were selected as they jointly evaluate the core outcome set requirements. ${ }^{56}$ Details of the secondary outcome measures and rationale for selection are found in table 1.

\section{Primary outcome measures}

- Overall pain, Numerical Rating Scale (NRS): The NRS is a unidimensional 11-point scale (0-10) used to measure pain intensity, where 0 represents no pain and 10 represents maximum pain (eg, the worse pain you can possibly imagine) ${ }^{57}$ Patients with pain have been shown to prefer the NRS over other pain measure including the pain Visual Analogue Scale owing to simplicity and clarity. ${ }^{5758}$ The NRS has demonstrated good reliability, validity and responsiveness and has been used extensively in pain research. ${ }^{59-61}$ A reduction of 2.5 points on the NRS has been shown to be clinically important for chronic LBP. ${ }^{60-62}$ Participants will score pain in three categories: 'worst pain over the last 2 weeks', 'least pain over the last 2 weeks' and 'average pain level today'.

- RMDQ: The RMDQ is one of the most widely used outcome measures for LBP, with well-established good levels of validity and reliability. ${ }^{63}$ The RMDQ has 
been selected over its counterparts owing to its superior measurement properties in patients reporting moderate disability demonstrated by those stratified into the medium risk group by the STarT Back Tool. $^{76263}$ The 24-item questionnaire takes approximately 5 min to complete and includes items assessing physical activity, sleep, psychosocial factors, activities of daily living, appetite and pain. ${ }^{64}$ Scores range from 0 (no disability) to 24 (maximum disability), with a change of 3.5 points deemed clinically significant. ${ }^{62}$

\section{Sample size}

As the number of FCP physiotherapists that are registered to prescribe is currently limited, ${ }^{65}$ three first contact APPs $(n=3)$, across three primary care sites representative of English geography (x1 capitol city, x1 regional city, $\mathrm{x} 1$ rural town), will recruit, assess and treat $\mathrm{n}=10$ participants per APP, to enable the evaluation of recruitment rates across clinicians and the feasibility of the trial methods in both metropolitan and rural healthcare services. ${ }^{27} 4344$ This feasibility trial does not aim to estimate the sample size required for the full trial as feasibility trials for cRCTs have been shown not to adequately predict sample size, therefore large numbers of participants are not required. ${ }^{436}$ A total sample of $n=30$ patients will be recruited as a sample size of $n>20$ is regarded as adequate when testing feasibility objectives for cRCTs. ${ }^{27} 284344$ This allows for some loss to follow-up of participants.

\section{Data analysis}

A CONSORT diagram will be used to describe the flow of participants and lost to follow-up rates. This will be used to analyse feasible eligibility, recruitment and follow-up rates. ${ }^{30}$ Only data from fully completed outcome questionnaires will be included in the data analysis; however, the number of partly completed outcome questionnaires will be noted and reasons for this explored in the embedded qualitative component of the trial. Data will be tabulated, and primary descriptive analysis of the data will be completed to test procedure. ${ }^{27}{ }^{45}{ }^{46}$ Causality will not be statistically analysed as this is not within the scope of this feasibility trial. ${ }^{45}$ The distribution of the scores on the RMDQ will be evaluated at baseline, 6 and 12 weeks following initial intervention. The percentage of scores equalling $0 / 24$ at 12 weeks will be used to measure a potential floor effect. ${ }^{67}$

\section{EMBEDDED QUALITATIVE COMPONENT \\ Design}

An embedded qualitative component will be used as recommended by current guidance, to address trial objectives and to refine and adapt the proposed full trial design following evaluation. ${ }^{68} 69$ The methodology was designed and is reported using the Consolidated Criteria for Reporting Qualitative Health Research. ${ }^{70}$

\section{Advanced physiotherapy practitioners}

Semistructured in-depth face-to-face interviews with all of the APPs $(n=3)$ will be used to evaluate their views and experiences about the feasibility, suitability and acceptability of the trial, specifically evaluating trials objectives. ${ }^{26-29} 7172$ Interviews will be undertaken by one researcher (TN) following completion of participant data collection, to evaluate the research objectives and to gather qualitative data regarding the participants' views, perceptions and experiences about taking part, future risks and how the trial might be improved. ${ }^{27}{ }^{28}$ Question design was informed by the methodological literature and developed by a team of experts in the fields of physiotherapy, primary care, NMP, health policy and trial methodology. ${ }^{55}$ A patient and public involvement group reviewed the questions for appropriateness and clarity. ${ }^{73}$ Prior to completing the interviews, the APP participants will be provided with an information sheet and will have the opportunity to ask the researcher any questions about the interview process. Consent to taking part will be gained using a consent form. Interviews will be recorded and transcribed verbatim. Transcripts will be returned to participants for inspection, comments and corrections prior to analysis, to ensure all views and thoughts are captured. ${ }^{71}$

\section{Patients}

A focus group of patients will take place following the 12 weeks assessment point, specifically to evaluate the research objectives. ${ }^{27} 74$ Focus groups are recognised to produce data on collective views, generating a rich understanding of participants' experiences. ${ }^{75}$ A purposive sample of six to eight patients, representative of ages and sexes will be used; this sample size is reported in the literature as the optimum. ${ }^{74}$ The focus group will meet in the qualitative laboratory within the CPR Spine at the University of Birmingham, UK, ensuring confidentiality. The focus group will be conducted by two researchers (facilitator and observer) using a predetermined topic guide designed to assess the research objectives, developed by a team of experts in the fields of physiotherapy, primary care, NMP, health policy and trial methodology and informed by the methodological literature. ${ }^{45} 55$ The topic guide has been reviewed by a patient and public involvement group to ensure appropriateness and clarity. ${ }^{73}$ Consent to participate in the focus group will be taken prior to the focus group commencing. The participants will receive an information leaflet and have the opportunity to have any questions answered by the researchers. The focus group will be recorded and transcribed verbatim. Transcripts will be returned to participants for comments/correction to ensure all views are represented. ${ }^{70}$

\section{Analysis and findings}

To fulfil the trial objectives, a thematic analysis approach will be used to analyse and synthesise the qualitative data. ${ }^{45} 7677$ This systematic, inductive and interactive 
method is recognised to be useful in identifying the key thoughts and views of the population being studied. The method is useful where there are likely to be both similarities and diversity of opinion and where the intervention is novel, often providing explanations alluding to how the concerns may be resolved or processed in preparation for a full trial. ${ }^{76-79}$ Focus group and interview transcripts will be coded line-by-line using NVivo 11 software (QSR International, Melbourne, Australia) by one researcher (TN) and be verified by a second researcher (AR). ${ }^{46} 7778$ Rigorous comparative analysis will be completed by one researcher $(\mathrm{TN})$ to identify similarities and differences within the data, informing the development of descriptive categories which will be linked, merged or split to synthesise a conceptual understanding of the data. ${ }^{778}$ To avoid single researcher bias, a second researcher (AR) will re-interrogate the data to validate or contradict findings. ${ }^{77}$ Outcomes will then be discussed with a panel of experts for confirmation and agreement. ${ }^{767779}$

\section{Integration: feasibility, suitability and acceptability}

Following data analysis of the trial and embedded qualitative components, the quantitative and qualitative data will be assessed against a success criterion outlined a priori (table 2). The predetermined success criteria were developed by a team of experts in the fields of physiotherapy, primary care, NMP, health policy and trial methodology and informed by the methodological literature. ${ }^{45} 5580$ Trial objectives will be considered successful if the success criteria are satisfied following the integration of the quantitative and qualitative findings. ${ }^{80}$

\section{Patient and public involvement}

Patients with LBP are part of our research team/co-investigators to ensure that the patient perspective is central. There is a PPI representative on both the Trial Management Group and Trial Steering Group to ensure that patients and the public are involved at all steps in the research process.

Patients have contributed to the development of the interview/focus group questions, participant information sheet, consent form, and importantly to the processes of data analysis and interpretation and producing a lay summary of findings. They have reviewed this protocol and have helped to ensure that their involvement is fully considered.

\section{Data storage}

All data will be electronic and stored in password-protected computer files that can be accessed only by study investigators at the University of Birmingham. Participants who choose to disclose personal details will be additionally protected via coding on data files. This coding will be kept in a password protected computer file on the University of Birmingham server, only accessible to the research team ensuring confidentiality. ${ }^{45} 81$ These personal data and participant contact details (stored during study to arrange focus groups and interviews) will be securely
Table 2 Success criteria

\begin{tabular}{|c|c|}
\hline General objectives & Success criteria \\
\hline \multirow[t]{2}{*}{ Eligibility criteria } & $\begin{array}{l}\text { A favourable number of patients fit } \\
\text { the eligibility criteria to enable the } \\
\text { stipulated recruitment rate }\end{array}$ \\
\hline & $\begin{array}{l}\text { APPs agreed with the eligibility } \\
\text { criteria }\end{array}$ \\
\hline \multirow[t]{2}{*}{ Recruitment strategy } & $\begin{array}{l}\text { Participants were recruited within the } \\
\text { time constraints of the local clinical } \\
\text { environment }\end{array}$ \\
\hline & $\begin{array}{l}\text { Patients and APPs report that they } \\
\text { were happy with the recruitment } \\
\text { strategy }\end{array}$ \\
\hline \multirow[t]{3}{*}{ Data collection methods } & $\begin{array}{l}\text { Data were collected with ease via } \\
\text { REDCap and no complications were } \\
\text { experienced }\end{array}$ \\
\hline & Data completeness of $\geq 80 \%$ \\
\hline & $\begin{array}{l}\text { Patients and APPs report that they } \\
\text { were happy with the data collection } \\
\text { methods }\end{array}$ \\
\hline \multirow[t]{3}{*}{ Follow-up procedures } & $\begin{array}{l}100 \% \text { of participants were contacted } \\
\text { for follow-up }\end{array}$ \\
\hline & $\begin{array}{l}\geq 80 \% \text { completion of follow-up } \\
\text { outcome measures }\end{array}$ \\
\hline & $\begin{array}{l}\text { Patients and APPs report that } \\
\text { they were happy with follow-up } \\
\text { procedures }\end{array}$ \\
\hline
\end{tabular}

\begin{tabular}{l}
\hline Specific objectives \\
Feasibility \\
Participant recruitment \\
rates
\end{tabular}

Ease of fitting accelerometers

\section{Success criteria}

Recruitment target of $n=10$ per clinician met in the time available (3months)

the allocated clinical time allowed
with the FCP APP
Patients and APPs report that
accelerometers were fitted with no
issues

Accelerometer data collection

REDCap was able to capture the data from the accelerometers with no errors or data loss

Patients report that they were happy with data collection using accelerometers/burden within subjectively appropriate limits

Capacity (time and effort) APPs report that adequate time of clinicians' complete trial- was allowed to complete all tasks related tasks required by them during the trail

Training required by clinicians

\section{APPs report that they had adequate} training to be able to complete the tasks required by them during the trial

\begin{tabular}{|c|c|}
\hline \multicolumn{2}{|l|}{ Suitability } \\
\hline \multirow[t]{2}{*}{ Outcome measures } & Data completeness of $\geq 80 \%$ \\
\hline & $\begin{array}{l}\text { Patients and APPs report that the } \\
\text { outcome measures were appropria } \\
\text { and self-explanatory }\end{array}$ \\
\hline $\begin{array}{l}\text { Compliance with wearing } \\
\text { the accelerometers }\end{array}$ & $\begin{array}{l}\text { Data collected } \geq 80 \% \text { of the } \\
\text { requested time ( } 16 \text { hours/day for } \\
7 \text { days) }\end{array}$ \\
\hline
\end{tabular}

Continued 


\begin{tabular}{|c|c|}
\hline Specific objectives & Success criteria \\
\hline $\begin{array}{l}\text { Time required to conduct } \\
\text { each stage of the protocol }\end{array}$ & $\begin{array}{l}\text { APPs report having adequate time to } \\
\text { complete each stage of the protocol }\end{array}$ \\
\hline \multirow[t]{2}{*}{ Service infrastructure } & $\begin{array}{l}\text { Recruitment targets met } \\
\text { Data completeness of } \geq 80 \%\end{array}$ \\
\hline & $\begin{array}{l}\text { APPs report that adequate service } \\
\text { infrastructure is in place to allow for } \\
\text { a full trial to be completed }\end{array}$ \\
\hline \multicolumn{2}{|l|}{ Acceptability } \\
\hline Intervention & $\begin{array}{l}\text { Patients and APPs report that } \\
\text { the intervention was appropriate/ } \\
\text { satisfactory }\end{array}$ \\
\hline
\end{tabular}

APPs, advanced physiotherapy practitioners; FCPs, first contact advanced physiotherapy practitioners.

destroyed at the end of the study. No participants will be identifiable in data presentation or dissemination. The confidentiality of data will be preserved when the data are transmitted to sponsors and co-investigators by maintaining the de-personalised data format and ensuring that no data are traceable to an individual participant. The password-protected files will be retained for 10 years, in a confidential, locked storage unit, satisfying university code of practice.

\section{ETHICS AND DISSEMINATION \\ Ethical considerations}

The feasibility trial will be conducted in accordance with the principles of the Research Governance Framework for Health and Social Care. To ensure that the study is conducted in an ethical manner within best research practice, Health Research Authority (HRA) ethical approval was sought via the Integrated Research Application System (IRAS) ID 250734. ${ }^{4581}$ Approval was granted on 30 October 2018. Participants' inclusion within the study will be entirely voluntary, with no incentives offered to participants to minimise bias. ${ }^{45}{ }^{46}$ Participant consent will be gained using an online consent form following the provision of information explaining the rationale, content and research dissemination plans to ensure ethical recruitment of participants. ${ }^{45} 81$ Participants will be free to withdraw at any time. ${ }^{45} 46$

\section{Dissemination of findings}

The study's findings will be disseminated via study reports, publication in academic peer-reviewed journals and conference presentations. ${ }^{45} 46$ The results will be communicated to participants as a summary report written in lay language including key findings and plans for future research.

\section{DISCUSSION}

The results from this prospective, mixed-methods, singlegroup feasibility trial with an embedded qualitative component will serve to inform researchers about the feasibility, suitability and acceptability of the specific methods evaluated, in preparation for a full RCT to assess the clinical and cost-effectiveness of physiotherapist prescribing for LBP in primary care. Evidence is required by researchers, policy-makers and health-service managers to inform decisions regarding the selection of appropriate, rigorous, clinically safe and economically sound design of a robust, high-quality full RCT with low risk of bias. It is anticipated that the results of this study will be used in conjunction with ethical evaluation, economic and risk analyses, as well as consultation with key stakeholders including the British health consumer when contemplating change, enhancement or redesign of the essential full RCT.

Contributors TDN is a clinical advanced practice physiotherapist and $\mathrm{PhD}$ candidate at the University of Birmingham (UK). ABR is a reader in musculoskeletal rehabilitation sciences and lead supervisor. JFM is a professor of clinical pharmacy and co-supervisor. Both supervisors ensured the rigour of methods and analyses. All authors have contributed to the content of this article. TDN wrote the first draft of this article and has worked with all authors to develop subsequent drafts. All authors gave final approval prior to publication. Patients and the general public were involved in the design of this study via PPI evaluation groups.

Funding Health Education England funding has allowed for the procurement of accelerometers and the associated IT programmes to ensure that innovative physical measures can be evaluated alongside patient-reported outcome measures. The Private Physiotherapy Educational Fund has allowed for the procurement of $x 3$ tablet computers for use in data collection and 7.5 hours per week of the principal Investigators time for 18 months.

Competing interests None declared.

Patient consent for publication Not required.

Ethics approval This trial is approved by the Health Research Authority (HRA). Ethical approval was sought via the Integrated Research Application System (IRAS) ID 250734.

Provenance and peer review Not commissioned; externally peer reviewed.

Open access This is an open access article distributed in accordance with the Creative Commons Attribution Non Commercial (CC BY-NC 4.0) license, which permits others to distribute, remix, adapt, build upon this work non-commercially, and license their derivative works on different terms, provided the original work is properly cited, appropriate credit is given, any changes made indicated, and the use is non-commercial. See: http://creativecommons.org/licenses/by-nc/4.0/.

\section{REFERENCES}

1. Campbell J, Colvin LA. Management of low back pain. BMJ 2013;347:bmj.f3148.

2. Macfarlane GJ, Beasley M, Jones EA, et al. The prevalence and management of low back pain across adulthood: results from a population-based cross-sectional study (the MUSICIAN study). Pain 2012;153:27-32.

3. Bernstein IA, Malik Q, Carville S, et al. Low back pain and sciatica: summary of NICE guidance. BMJ 2017;356:i6748.

4. HSE. Work-related Musculoskeletal Disorder (WRMSDs) Statistics, Great Britain. London: Health and Safety Executive, 2016.

5. Parsons S, Ingram M, Clarke-Cornwell A, et al. A heavy burden: the occurrence and impact of musculoskeletal conditions in the United Kingdom today. 2011.

6. Cherkin D, Balderson B, Brewer G, et al. Evaluation of a riskstratification strategy to improve primary care for low back pain: the MATCH cluster randomized trial protocol. BMC Musculoskelet Disord 2016;17:361.

7. Hill JC, Whitehurst DG, Lewis M, et al. Comparison of stratified primary care management for low back pain with current best practice (STarT Back): a randomised controlled trial. Lancet 2011;378:1560-71. 
8. NICE. Low back pain and sciatica in over 16s: assessment and management (NICE guideline NG59): Full guideline.: National Institute for Health and Care Excellence, 2016.

9. NHS-Digital. General and personal medical services, England As at 31 March 2017. London, UK: NHS Digital, 2017.

10. Majeed A. Shortage of general practitioners in the NHS. BMJ 2017;358:j3191.

11. NHS-England. INNOVATION INTO ACTION: supporting delivery of the NHS five year forward view. London, UK: NHS England, 2015.

12. NHS-England. Allied health professions into action: using allied health professionals to transform health, care and wellbeing. London, UK: NHS England, 2017.

13. CSP. Scope of Practice London: Chartered Society of Physiotherapy. 2016 (Accessed 3rd Dec 2017).

14. Suckley J. Core Clinical Competencies for Extended-scope Physiotherapists Working in Musculoskeletal (MSK) interface clinics based in primary care. Salford: A delphi consensus study University of Salford, 2012.

15. Saxon RL, Gray MA, Oprescu FI. Extended roles for allied health professionals: an updated systematic review of the evidence. $J$ Multidiscip Healthc 2014;7:479.

16. Stanhope J, Grimmer-Somers K, Milanese S, et al. Extended scope physiotherapy roles for orthopedic outpatients: an update systematic review of the literature. $J$ Multidiscip Healthc 2012:5:37-45.

17. NHS-England. General Practice Forward View. London, UK: NHS England, 2015.

18. HEE. Musculoskeletal clinical fellowship 2017/18 - role outline. London: Health Education England, 2017.

19. CSP. General practice physiotherapy posts: a guide for implementation and evaluation in England. London: CSP, RCGP, BMA, 2017

20. Schneider GM, Jull G, Thomas K, et al. Screening of patients suitable for diagnostic cervical facet joint blocks-a role for physiotherapists. Man Ther 2012;17:180-3.

21. Rabey M, Morgans S, Barrett C. Orthopaedic physiotherapy practitioners: surgical and radiological referral rates. Clinical Governance: An International Journal 2009;14:15-19.

22. Carey N, Stenner K, Gage H. Evaluation of physiotherapist and podiatrist independent prescribing, mixing of medicines, and prescribing of controlled drugs. London: Policy Research Programme, Department of Health, 2016.

23. Noblet T, Marriott J, Graham-Clarke E, et al. Barriers to and facilitators of independent non-medical prescribing in clinical practice: a mixed-methods systematic review. J Physiother 2017:63:221-34.

24. Smith P, Mossialos E, Papanicolas I. Performance measurement for health system improvement: experiences, challenges and prospects. Copenhagen, Denmark: WHO, 2008.

25. Noblet T, Marriott J, Graham-Clarke E, et al. Clinical and costeffectiveness of non-medical prescribing: A systematic review of randomised controlled trials. PLoS One 2018;13:e0193286.

26. Bowen DJ, Kreuter M, Spring B, et al. How we design feasibility studies. Am J Prev Med 2009;36:452-7.

27. Tickle-Degnen L. Nuts and bolts of conducting feasibility studies. Am J Occup Ther 2013;67:171-6.

28. Shanyinde M, Pickering RM, Weatherall M. Questions asked and answered in pilot and feasibility randomized controlled trials. BMC Med Res Methodol 2011;11:117.

29. Lancaster GA, Dodd S, Williamson PR. Design and analysis of pilot studies: recommendations for good practice. J Eval Clin Pract 2004;10:307-12.

30. Eldridge SM, Chan CL, Campbell MJ, et al. CONSORT 2010 statement: extension to randomised pilot and feasibility trials. Pilot Feasibility Stud 2016;2:64

31. Eldridge S, Bond C, Campbell M, et al. Definition and reporting of pilot and feasibility studies. Trials 2013;14.

32. Schulz KF, Altman DG, Moher D. CONSORT 2010 Statement: updated guidelines for reporting parallel group randomised trials. BMC Med 2010;8:18.

33. Staniszewska S, Brett J, Simera I, et al. GRIPP2 reporting checklists: tools to improve reporting of patient and public involvement in research. BMJ 2017;358:j3453.

34. Staniszewska S, Brett J, Simera I, et al. GRIPP2 reporting checklists: tools to improve reporting of patient and public involvement in research. Res Involv Engagem 2017;3:13.

35. The Joanna Briggs Institute. The joanna briggs institute reviewers manual 2014 methodology for jbi umbrella reviews. Adelaide: The Joanna Briggs Institute, 2014.
36. Sandelowski M, Voils Cl, Barroso J. Defining and designing mixed research synthesis studies. Res Sch 2006;13:29.

37. Heyvaert M, Maes B, Onghena P. Mixed methods research synthesis: definition, framework, and potential. Qual Quant 2013:47:659-76.

38. Barker D, McElduff P, D'Este C, et al. Stepped wedge cluster randomised trials: a review of the statistical methodology used and available. BMC Med Res Methodol 2016;16:69.

39. Prost A, Binik A, Abubakar I, et al. Logistic, ethical, and political dimensions of stepped wedge trials: critical review and case studies. Trials 2015;16:351

40. Woertman W, de Hoop E, Moerbeek M, et al. Stepped wedge designs could reduce the required sample size in cluster randomized trials. J Clin Epidemiol 2013;66:752-8.

41. Beard E, Lewis JJ, Copas A, et al. Stepped wedge randomised controlled trials: systematic review of studies published between 2010 and 2014. Trials 2015;16:353.

42. Mdege ND, Man MS, Taylor Nee Brown CA, et al. Systematic review of stepped wedge cluster randomized trials shows that design is particularly used to evaluate interventions during routine implementation. J Clin Epidemiol 2011;64:936-48.

43. Kristunas CA, Hemming K, Eborall $\mathrm{HC}$, et al. The use of feasibility studies for stepped-wedge cluster randomised trials: protocol for a review of impact and scope. BMJ Open 2017;7:e017290.

44. Arain $\mathrm{M}$, Campbell MJ, Cooper $\mathrm{CL}$, et al. What is a pilot or feasibility study? A review of current practice and editorial policy. BMC Med Res Methodol 2010;10:67.

45. Hicks CM. Research methods for clinical therapists: applied project design and analysis: Elsevier Health Sciences, 2009.

46. Bowling A. Research methods in health: investigating health and health services. UK: McGraw-Hill Education, 2014.

47. NICE. Clinical Knowledge Summaries: Back pain - low (without radiculopathy. London, UK: NICE, 2017. (Accessed 4th Mar 2018).

48. Henschke N, Maher CG, Refshauge KM, et al. Prognosis in patients with recent onset low back pain in Australian primary care: inception cohort study. BMJ 2008;337:a171.

49. Abbott JH, Mercer $\mathrm{S}$. The natural history of acute low back pain. New Zealand Journal of Physiotherapy 2002;30:8-17.

50. REDCap. REDCap: Research Electronic Data Capture2018. https:// projectredcap.org (Accessed 4th Mar 2018).

51. Wright KB. Researching internet-based populations: advantages and disadvantages of online survey research, online questionnaire authoring software packages, and web survey services. Journal of Computer-Mediated Communication 2005;10:00.

52. Fritz JM, Beneciuk JM, George SZ. Relationship between categorization with the STarT Back Screening Tool and prognosis for people receiving physical therapy for low back pain. Phys Ther 2011;91:722-32.

53. CSP. Treatment for back pain. London, UK: Chartered Society of Physiotherapy, 2012.

54. APA. The Physiotherapy Prescribing Pathway: Proposal for the endorsement of registered physiotherapists for autonomous prescribing. Melbourne, Australia: APA, 2015

55. O'Cathain A, Hoddinott P, Lewin S, et al. Maximising the impact of qualitative research in feasibility studies for randomised controlled trials: guidance for researchers. Pilot Feasibility Stud 2015;1:32.

56. Chiarotto A, Deyo RA, Terwee CB, et al. Core outcome domains for clinical trials in non-specific low back pain. Eur Spine $J$ 2015;24:1127-42

57. Hawker GA, Mian S, Kendzerska T, et al. Measures of adult pain: Visual Analog Scale for Pain (VAS Pain), Numeric Rating Scale for Pain (NRS Pain), McGill Pain Questionnaire (MPQ), Short-Form McGill Pain Questionnaire (SF-MPQ), Chronic Pain Grade Scale (CPGS), Short Form-36 Bodily Pain Scale (SF). Arthritis Care Res 2011;63:S240-52.

58. de C Williams AC, Davies HT, Chadury Y. Simple pain rating scales hide complex idiosyncratic meanings. Pain 2000;85:457-63.

59. Ferraz MB, Quaresma MR, Aquino LR, et al. Reliability of pain scales in the assessment of literate and illiterate patients with rheumatoid arthritis. J Rheumatol 1990;17:1022-4.

60. Farrar JT, Young JP, LaMoreaux L, et al. Clinical importance of changes in chronic pain intensity measured on an 11-point numerical pain rating scale. Pain 2001;94:149-58.

61. Childs JD, Piva SR, Fritz JM. Responsiveness of the numeric pain rating scale in patients with low back pain. Spine 2005;30:1331-4.

62. Ostelo RW, de Vet HC. Clinically important outcomes in low back pain. Best Pract Res Clin Rheumatol 2005;19:593-607.

63. Stevens ML, Lin CC, Maher CG. The roland morris disability questionnaire. J Physiother 2016;62:116.

64. Roland M, Fairbank J. The roland-morris disability questionnaire and the oswestry disability questionnaire. Spine 2000;25:3115-24. 
65. CSP, RCGP, BMA. First contact physiotherapy posts in general practice: a guide for implementation in England. 3 edn. London, UK: CSP, RCGP, BMA, 2018.

66. Eldridge SM, Costelloe $\mathrm{CE}$, Kahan BC, et al. How big should the pilot study for my cluster randomised trial be? Stat Methods Med Res 2016;25:1039-56.

67. Lim CR, Harris K, Dawson J, et al. Floor and ceiling effects in the OHS: an analysis of the NHS PROMs data set. BMJ Open 2015;5:e007765.

68. Craig P, Dieppe P, Macintyre S, et al. Developing and evaluating complex interventions: the new medical research council guidance. BMJ 2008;337:337:a1655.

69. O'Cathain A, Goode J, Drabble SJ, et al. Getting added value from using qualitative research with randomized controlled trials: a qualitative interview study. Trials 2014;15:15.

70. Tong A, Sainsbury P, Craig J. Consolidated criteria for reporting qualitative research (COREQ): a 32-item checklist for interviews and focus groups. Int J Qual Health Care 2007;19:349-57.

71. Jamshed S. Qualitative research method-interviewing and observation. J Basic Clin Pharm 2014;5:87.

72. Galletta A. Mastering the semi-structured interview and beyond: from research design to analysis and publication: NYU press. 2013

73. Brett J, Staniszewska S, Mockford C, et al. Mapping the impact of patient and public involvement on health and social care research: a systematic review. Health Expect 2014;17:637-50.

74. Bloor M. Focus groups in social research: Sage, 2001.

75. Gill P, Stewart K, Treasure E, et al. Methods of data collection in qualitative research: interviews and focus groups. Br Dent $J$ 2008;204:291-5

76. Jones M, Alony I. Guiding the use of grounded theory in doctoral studies-an example from the australian film industry. 2011.

77. Charmaz K, Belgrave L. Qualitative interviewing and grounded theory analysis: The SAGE handbook of interview research: The complexity of the craft, 2012:347-65.

78. Burck C. Comparing qualitative research methodologies for systemic research: the use of grounded theory, discourse analysis and narrative analysis. J Fam Ther 2005;27:237-62.

79. Corbin J, Strauss A. Basics of qualitative research: techniques and procedures for developing grounded theory. Thousand Oaks, 2008.

80. Stow R, Ives N, Smith C, et al. A cluster randomised feasibility trial evaluating nutritional interventions in the treatment of malnutrition in care home adult residents. Trials 2015;16:433.

81. Kelley K, Clark B, Brown V, et al. Good practice in the conduct and reporting of survey research. Int J Qual Health Care 2003;15:261-6.

82. Koes BW, van Tulder M, Lin CW, et al. An updated overview of clinical guidelines for the management of non-specific low back pain in primary care. Eur Spine J 2010;19:2075-94.

83. de Graaf I, Prak A, Bierma-Zeinstra S, et al. Diagnosis of lumbar spinal stenosis: a systematic review of the accuracy of diagnostic tests. Spine 2006;31:1168-76.
84. Fraser S, Roberts L, Murphy E. Cauda equina syndrome: a literature review of its definition and clinical presentation. Arch Phys Med Rehabil 2009;90:1964-8.

85. NICE. Decision making and mental capacity. London UK: NICE, 2018.

86. Janssen MF, Pickard AS, Golicki D, et al. Measurement properties of the EQ-5D-5L compared to the EQ-5D-3L across eight patient groups: a multi-country study. Qual Life Res 2013;22:1717-27.

87. Leeuw M, Peters ML, Wiers RW, et al. Measuring fear of movement/ (re)injury in chronic low back pain using implicit measures. Cogn Behav Ther 2007;36:52-64.

88. Roelofs J, Goubert L, Peters ML, et al. The tampa scale for kinesiophobia: further examination of psychometric properties in patients with chronic low back pain and fibromyalgia. Eur J Pain 2004;8:495-502.

89. Rhodes RE, Mark RS, Temmel CP. Adult sedentary behavior: a systematic review. Am J Prev Med 2012;42:e3-28.

90. Griffin DW, Harmon DC, Kennedy NM. Do patients with chronic low back pain have an altered level and/or pattern of physical activity compared to healthy individuals? A systematic review of the literature. Physiotherapy 2012;98:13-23.

91. Lin CW, McAuley JH, Macedo L, et al. Relationship between physical activity and disability in low back pain: a systematic review and meta-analysis. Pain 2011;152:607-13.

92. Campbell C. Measuring physical behaviour in physiotherapists and in people with chronic low back pain: Ulster University, 2017.

93. Chastin SF, Granat MH. Methods for objective measure, quantification and analysis of sedentary behaviour and inactivity. Gait Posture 2010;31:82-6.

94. Edwardson CL, Winkler EAH, Bodicoat DH, et al. Considerations when using the activPAL monitor in field-based research with adult populations. J Sport Health Sci 2017;6:162-78.

95. Kozey-Keadle S, Libertine A, Lyden K, et al. Validation of wearable monitors for assessing sedentary behavior. Med Sci Sports Exerc 2011;43:1561-7.

96. Alsaadi SM, McAuley JH, Hush JM, et al. Poor sleep quality is strongly associated with subsequent pain intensity in patients with acute low back pain. Arthritis Rheumatol 2014;66:1388-94.

97. Alsaadi SM, McAuley JH, Hush JM, et al. Prevalence of sleep disturbance in patients with low back pain. Eur Spine $J$ 2011;20:737-43.

98. Kaila-Kangas L, Kivimäki M, Härmä M, et al. Sleep disturbances as predictors of hospitalization for back disorders-a 28-year follow-up of industrial employees. Spine 2006;31:51-6.

99. Haack M, Scott-Sutherland J, Santangelo G, et al. Pain sensitivity and modulation in primary insomnia. Eur $J$ Pain 2012;16:522-33.

100. Skender S, Ose J, Chang-Claude J, et al. Accelerometry and physical activity questionnaires - a systematic review. BMC Public Health 2016;16:515. 